\title{
Adsorption of triclosan on single wall carbon nanotubes: A first principle approach
}

\author{
S.M. Castro ${ }^{a}$, A.B. Araújo $^{b}$, R.F.P. Nogueira ${ }^{c}$, S. Guerini ${ }^{a}, *$ \\ a Departamento de Física, Universidade Federal do Maranhão, 65080-805 SãoLuís, MA, Brazil \\ b Instituto Federal do Maranhão, Campus São Luis-Centro Histórico, 65010-500 SãoLuís, MA, Brazil

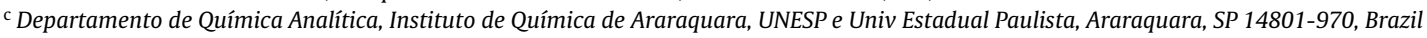

\section{A R T I C L E I N F O}

\section{Article history:}

Received 16 November 2016

Received in revised form 16 January 2017

Accepted 21 January 2017

Available online 24 January 2017

\section{Keywords:}

Nanotube

Triclosan

DFT

Electronic properties

\begin{abstract}
A B S T R A C T
The interaction of triclosan on $(8,0)$ and $(5,5)$ single wall carbon nanotube (SWCNT) was investigated using density functional calculations. The results show that the adsorption of triclosan modifies the electronic properties of pristine $(8,0)$ and $(5,5)$ SWCNT and induced changes in the electronic properties are dependent on the triclosan adsorption site. It was observed through binding energy that triclosan molecule interacts mainly via chemical process in parallel configuration to $(8,0)$ SWCNT, while interaction via physical process was observed with both $(8,0)$ and $(5,5)$ SWCNT. It is proposed that these SWCNTs are a potential filter device due to reasonable physical interaction with triclosan molecule. Furthermore, this type of filter could be reusable, therefore after the filtering, the SWCNTs could be separated from triclosan molecule.
\end{abstract}

(C) 2017 Elsevier B.V. All rights reserved.

\section{Introduction}

Endocrine disrupting compounds (EDCs) can mimic the biological activity of natural hormones in animals endocrine systems and impose a risk to animals and humans. Triclosan is a common antimicrobial found in personal care products, such as toothpastes, soaps, fabrics, and plastics [1,2]. Therefore, residual amounts are flushed into sewage streams and enters wastewater treatment plants resulting in contamination of surface water at $\mu \mathrm{g} \mathrm{L}^{-1}$ levels, and $\mathrm{mg} \mathrm{kg}^{-1}$ in sediments [3-5]. Although triclosan presents low toxicity, studies have demonstrated that triclosan can form highly toxic dioxin type derivatives under solar irradiation when present in surface water [6]. The molecular structure of triclosan contains phenolic hydroxyl group, which is electrochemically active and can be oxidized under proper conditions, so it is very important to develop a rapid, sensitive, and efficient method to remove triclosan.

Various removal methods have been used in water treatment such as adsorption on carbon nanomaterials [7,8], membrane separation [9], electrochemical or photochemical processes [10,11]. Another possibility for the removal or detection of these toxic substances in the environment is to use carbon nanostructures, which are now considered as possible filters or sensors for toxic

\footnotetext{
* Corresponding author.

E-mail address: silvete@gmail.com (S. Guerini).
}

substances. The hydrophobic surface of carbon nanostructures favors a strong interaction between organic pollutants and these nanostructures. Carbon nanostructures such as carbon nanotubes [12] present unique electronic and structural properties such as porous structure, large surface area, and thermostability, which favors the removal of organic contaminants from various aqueous systems [13].

In particular, the strong dependence of electronic properties of single wall carbon nanotubes (SWCNTs) on their atomic structure, such as nanotube diameter and chirality, provides a versatile range of applications. For example, SWCNTs could be used as gas sensors, field emission sources, polymer composite fillers, protein immobilizers, filters, electronic components. Furthermore, studies indicate that SWCNTs have high adsorption capacities of heavy metals [14], phenols [15], and organic substances [16].

Zaib et al. investigated the adsorption of bisphenol $A$ and $17 \beta$-estradiol onto SWCNTs and acid-treated SWCNTs at various temperatures by the use of several analytical techniques such as TEM, zeta potential measurements, and Raman spectra, confirmed the acidification of SWCNTs. SWCNTs and acid-treated SWCNTs proved to be ideal to adsorb bisphenol $A$ and $17 \beta$-estradiol [17].

In other work, Pan et. al. studied carbon nanostructures through adsorption and desorption (kinetic) experiments. The kinetic models used to analyze experimental data and obtain kinetic parameters indicated that SWCNTs have high potential for water 
treatment and purification, due to its high adsorption capacity, high adsorption rate, and strong retention of adsorbates [18].

In this study, the effects on the electronic properties of $(8,0)$ and $(5,5)$ SWCNT when interacting with triclosan molecule using ab initio simulations were investigated. SWCNT $(8,0)$ and $(5,5)$ show different electronic characteristics, the former is semiconductor while the latter is metallic. Currently the research by computer simulation is particularly important because the computational study of nanostructured systems constitutes an essential tool for scientific practice, able to make predictions of physicochemical properties of these systems and ensuring a better predicting and understanding of experimental results. Triclosan molecule have planar structure, which contributes to adsorption, and its fairly small molecule reduces the cost of computer simulation. The calculations predict that triclosan molecule interacts with nanotubes and the calculated binding energies are typical of an physical integration in most configurations considered.

\section{Method of calculation}

The theoretical calculations are based on first-principle spin polarized density functional theory [19] using numerical atomic orbitals as basis sets. We used the Siesta code [20], which performed full self consistent calculations solving the Kohn-Sham equations [21]. Double zeta basis set with polarization function [22] was used in all calculations. For the exchange and the correlation terms, local density approximation with the parameterization of Perdew and Wang [23] was used. The interaction between ionic cores and valence electrons is described by norm conserving pseudopotentials [24] in Kleinamn-Bylander form [25]. A cuttoff of 150 Ry for the grid integration was used to represent the charge density. The Brillouin zone is sampled with twenty three k-points special along the $\Gamma-X$ directions used Monkhorst-Pack method [26].

The calculations were performed using a $(8,0)$ and $(5,5)$ SWCNT, which are known in the literature by presents a behaves semiconductor and metallic character [27-29]. Periodic boundary conditions and a supercell approximation were used with a lateral approximation of $20 \AA$ A between nanotube center are ensure that the SWNT plus the molecule do not interact with the periodic images.
Table 1

Binding energies $\left(E_{b}\right)$, distance of binding $\left(D_{b}\right)$, gap energy $\left(E_{g}\right)$, and charge transfer (CT) calculated for different adsorption sites of $(8,0)$ SWCNT, as shown in Fig. 1. The minus and plus sign in the charge transfer values indicates that triclosan molecule receives or donates electronic charge, respectively.

\begin{tabular}{lllll}
\hline Configuration & $E_{b}(\mathrm{eV})$ & $D_{b}(\AA)$ & $E_{g}(\mathrm{eV})$ & $\mathrm{CT}\left(\mathrm{e}^{-}\right)$ \\
\hline (a) & 1.73 & 2.98 & 0.52 & +0.05 \\
(b) & 0.65 & 2.46 & 0.60 & -0.14 \\
(c) & 0.28 & 2.11 & 0.60 & -0.12 \\
(d) & 0.10 & 3.40 & 0.60 & +0.02 \\
(e) & 0.47 & 3.42 & 0.57 & -0.01 \\
\hline
\end{tabular}

The supercells that were used have $128 \mathrm{C}$ atoms to $(8,0)$ SWCNT and $180 \mathrm{C}$ atoms to $(5,5)$ SWCNT, respectively. On the other hand, the $(8,0)$ SWCNT have a diameter of $6.37 \AA$ and the $(5,5)$ SWCNT has a diameter of the $6.78 \AA$ Ahich implies that the two nanotubes have a very close curvature. The structural optimizations were performed using a conjugated gradient procedure, and the atomic positions of the structure are relaxed until all the force components are smaller than $0.04 \mathrm{eV} / \AA$.

The binding energies $E_{b}$ between nanotubes and triclosan molecule were calculated using the basis set superposition error (BSSE) [30]. This correction is done through the counterpoise method using "ghost" atoms, as the following equation:

$E_{b}=-\left\{E_{T}[S W C N T+t]-E_{T}\left[S W C N T+t_{\text {ghost }}\right]-E_{T}\left[S W C N T_{\text {ghost }}+t\right]\right\}$

where $E_{T}[S W C N T+t]$ is the total energy for the system with SWCNT nanotube plus triclosan molecule in different configurations. The subscript "ghost" corresponds to additional basis wave functions centered at the position of the triclosan molecule or the nanotube, but without any atomic potential.

\section{Results and discussion}

The interaction between the $(8,0)$ and $(5,5)$ SWCNT and triclosan molecule were analyzed different in configurations, such as the triclosan molecule parallel to the axis of the nanotube (Fig. 1(a)), $\mathrm{H}$ atoms of triclosan molecule interacting with nanotube (Fig. 1(b)),

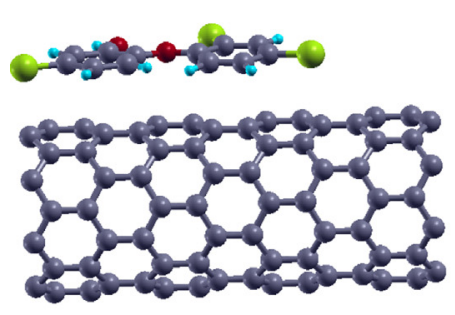

(a)

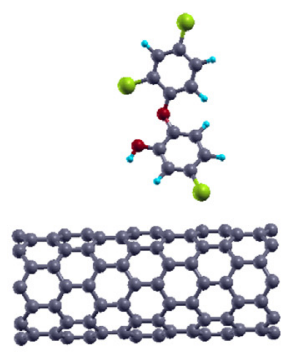

(d)

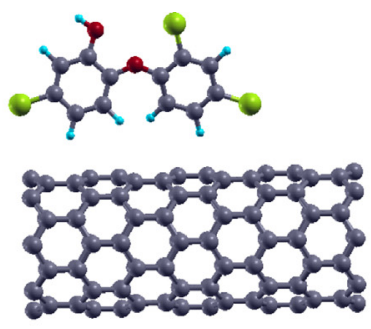

(b)

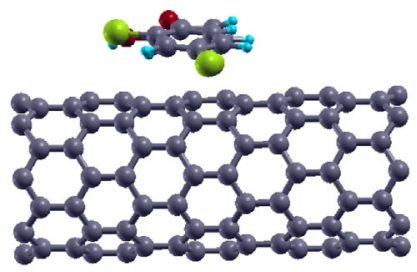

(e)

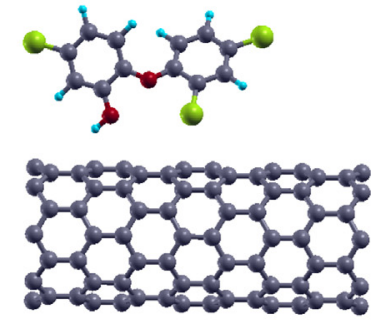

(c)

Fig. 1. Schematic view of all configurations considered for triclosan interaction with $(8,0)$ SWCNT to references. 

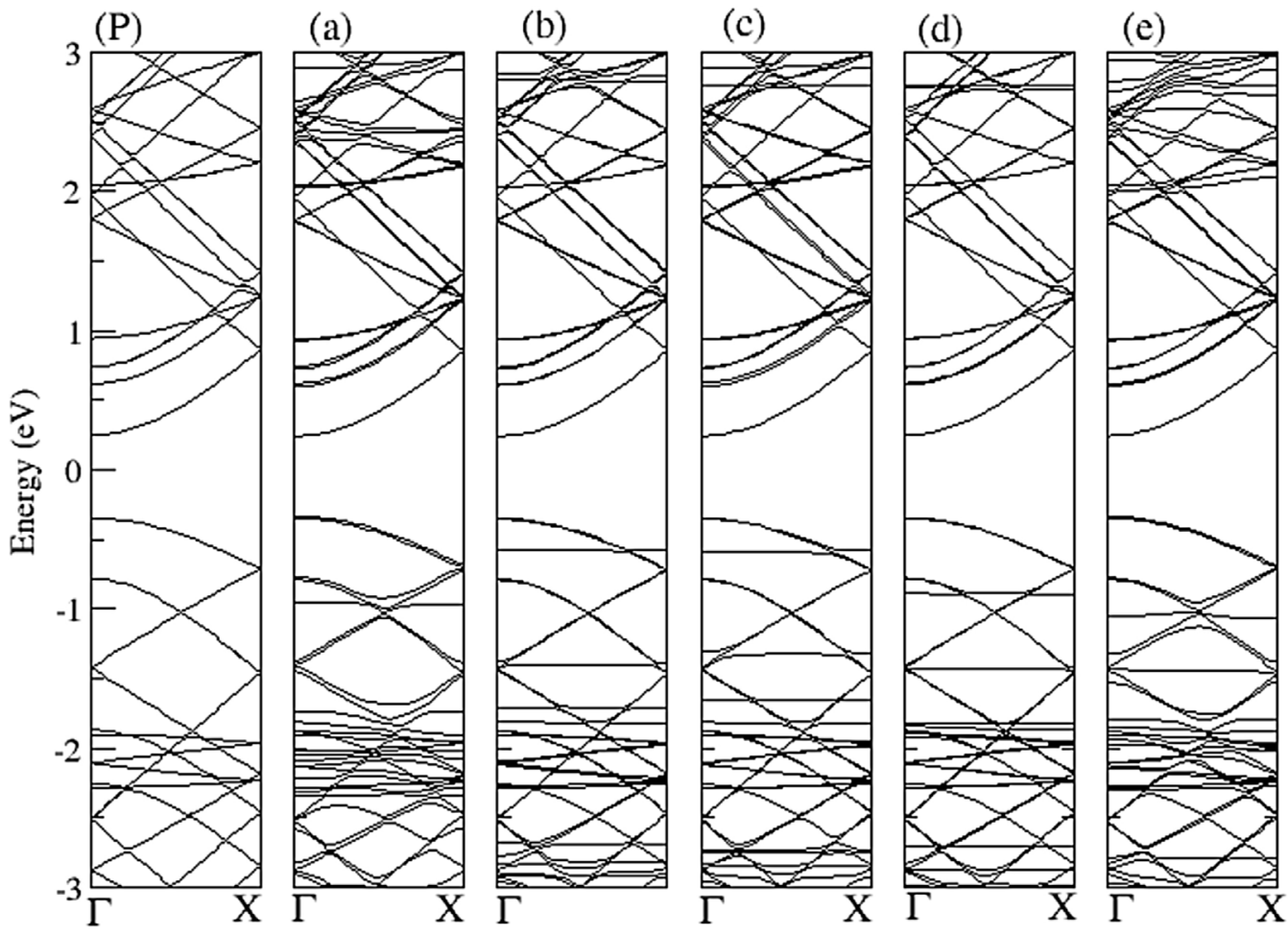

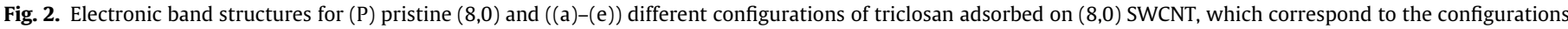
shown in Fig. 1(a)-(e), respectively. The Fermi energy is set to zero.

$\mathrm{O}$ atom and $\mathrm{Cl}$ atom interacting with nanotube (Fig. 1(c)), $\mathrm{Cl}$ atom interacting with nanotube (Fig. 1(d)), and triclosan molecule perpendicular to the tube axis (Fig. 1(e)). Fig. 1 shows optimized structures studied of $(8,0)$ SWCNT interacting with triclosan molecule. The configuration (a) (Fig. 1) was predicted to be the most stable, with a distance between SWCNT and triclosan molecule of about $2.98 \AA$. Table 1 , summarizes the calculated binding energy $\left(E_{b}\right)$ according to Eq. (1), distance of binding $\left(D_{b}\right)$, gap energy $E_{g}$ and the charge transfer for all the configurations studied in Fig. 1.

The analysis of results of the binding energies obtained for the different configurations pointed that the interaction between triclosan molecule and $(8,0)$ SWCNT occurs through physical or chemical process, depending on position of triclosan in relation to the nanotube. According to Machado et al. [31], binding energy values lower or equal to $0.83 \mathrm{eV}$ can be considered low, which corresponds to physical process, while energy above this value is considered high, characterizing a chemical process. The results showed that the most stable configuration is the one where the molecule is parallel to the tube axis, as can be seen from Fig. 1(a), presenting a binding energy of $1.73 \mathrm{eV}$. This results suggest that interaction between the triclosan molecule and nanotube depends strongly on the interaction between the aromatic rings of SWCNT and triclosan due to $\pi-\pi$ bond. The other configuration present

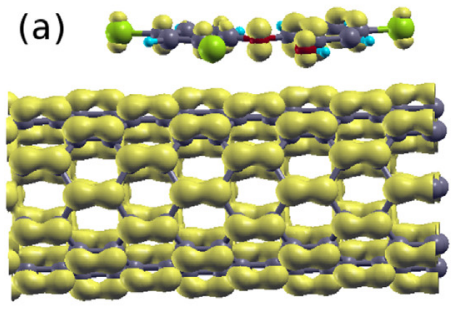

(b)
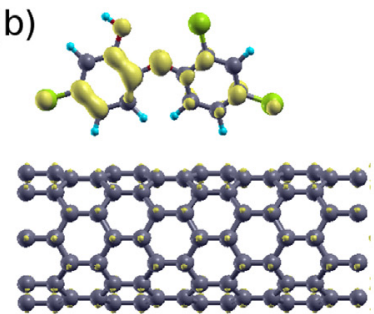

(c)
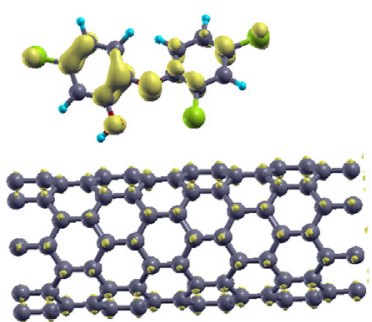

(d)<smiles>CC(C)(C)C1(C)CCCC1</smiles>

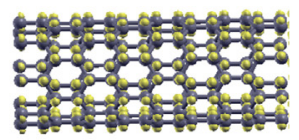

(e)

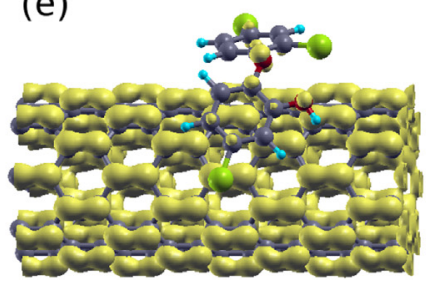

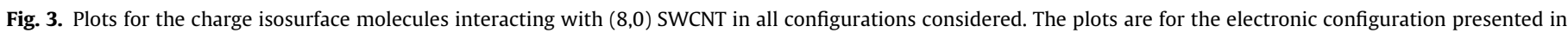
Fig. 2(a)-(e) around the Fermi level. The isovalue is 0.005 states/Ry. 


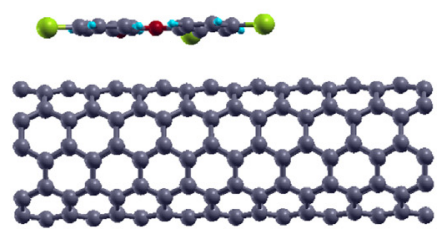

(a)

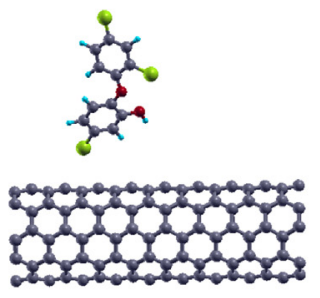

(d)
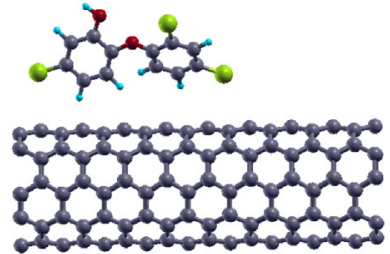

(b)

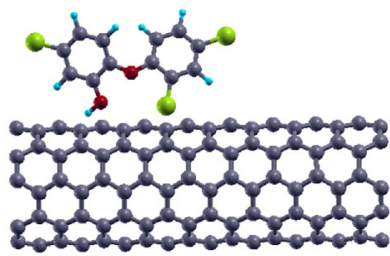

(c)

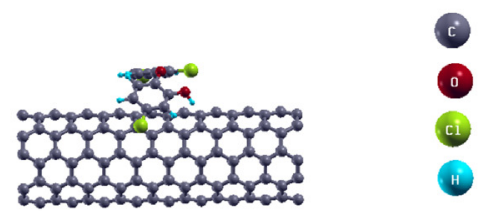

(e)

Fig. 4. Studied configurations for the triclosan molecule adsorbed on $(5,5)$ SWCNT.

low binding energy, i.e., $\leq 0.83 \mathrm{eV}$ indicating a physical process (Table 1).

The Mülliken population was analyzed and used for all studied configurations to predict the electronic charge transfer between SWCNT and triclosan molecule. It is important to point out that the Mülliken population does not supply a trustworthy number to charge transfer, but it indicates the trend and correct order of the charge transfer process. Triclosan molecule behaves as an electron acceptor or donor when interacting with the $(8,0)$ SWCNT, depending on the position of molecule. The triclosan molecule donates $0.05^{-e}$ to $(8,0)$ SWCNT in most stable configuration (Fig. 1(a)), while in configurations (b), (c) and (e) (Fig. 1) triclosan molecule receives $0.14^{-e}, 0.12^{-e}$ and $0.02^{-e}$, respectively, from $(8,0)$ SWCNT.
In configuration (d) triclosan molecule donates $0.01^{-e}$ to $(8,0)$ nanotube.

The calculated electronic band structures are presented for the pristine $(8,0)$ SWCNT (Fig. $2(\mathrm{P}))$ and for triclosan adsorbed on $(8,0)$ SWCNT (Fig. 2(a)-(e)) in all configurations shown in Fig. 1(a)-(e). The Fermi level is located in zero. The pristine $(8,0)$ SWCNT is a semiconductor with a energy gap calculated as $0.60 \mathrm{eV}$. This result is in agreement with those reported in the literature [32]. The results indicated that the electronic properties of $(8,0)$ SWCNT remained unchanged in all configurations considered, a semiconductor with energy gap around $0.60 \mathrm{eV}$.

It can be observed in Fig. 2(a)-(e), compared directly with pristine nanotube, that changes in electronic properties of $(8,0)$ SWCNT

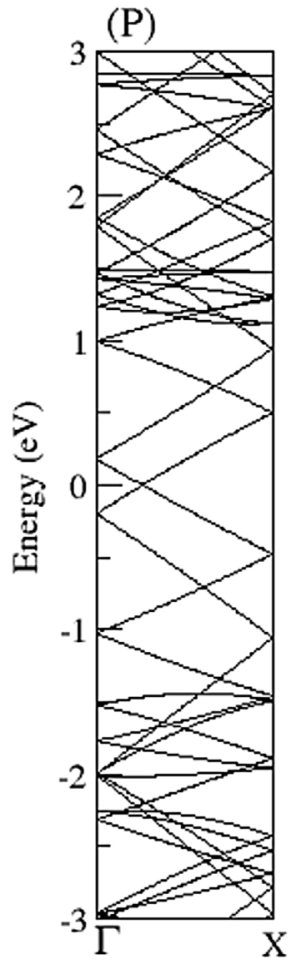

(a)

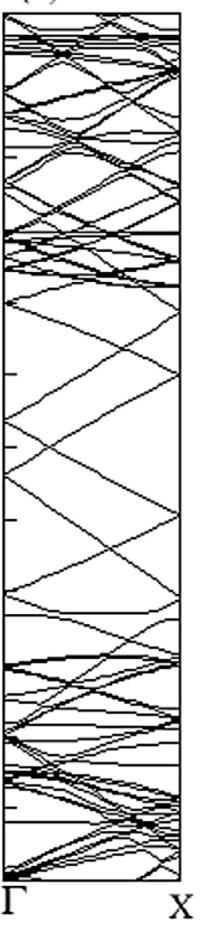

(b)

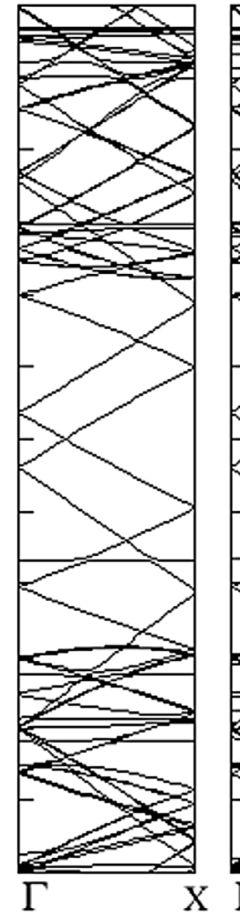

(c)

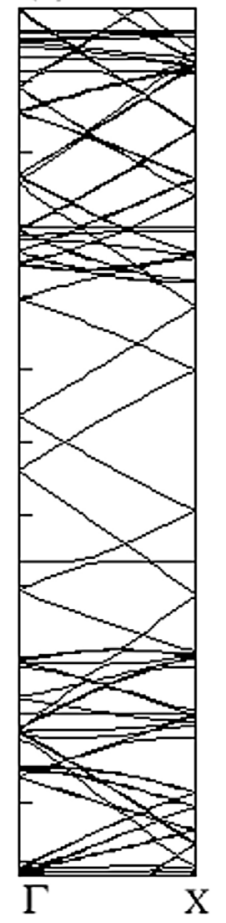

(d)

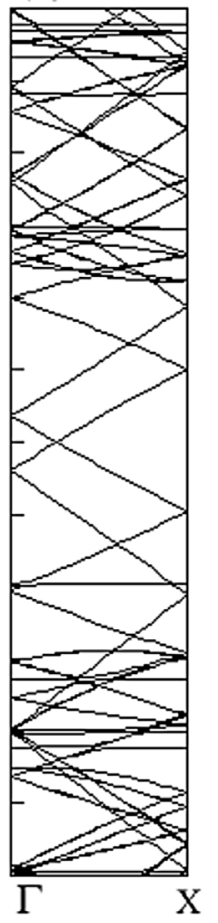

(e)

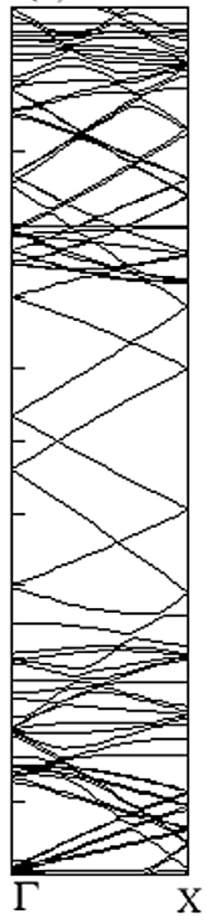

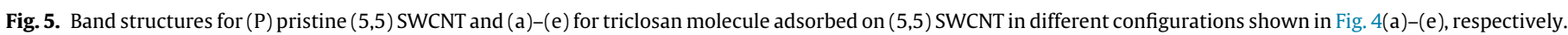
The Fermi level is localized in zero. 


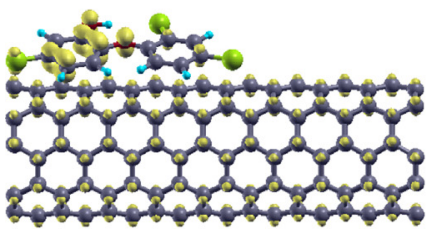

(a)

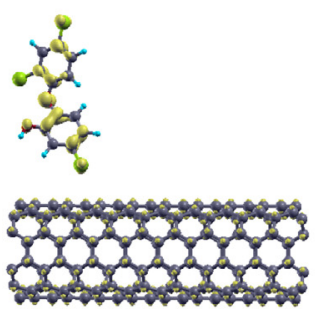

(d)

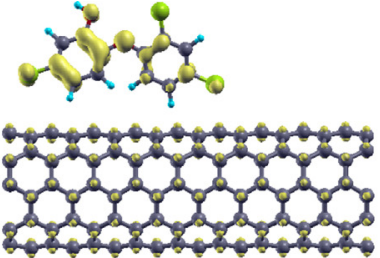

(b)

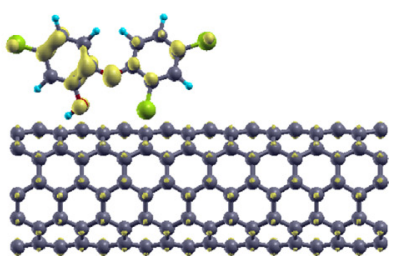

(c)

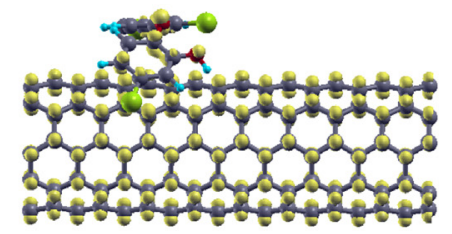

(e)

Fig. 6. Plots for the charge isosurface molecules interacting with $(5,5)$ SWCNT in all configurations considered. The plots are for the electronic configuration presented in Fig. 5(a)-(e) in region around $-1.07 \mathrm{eV}$ to $1.09 \mathrm{eV}$. The isovalue is 0.005 states/Ry.

occurred when interacting with triclosan, mainly in the valence band region. Fig. 2(a) shows the band structure for the most stable configuration (Fig. 1(a)) and it is possible to observe that there are mixtures between the levels of triclosan molecule with the nanotube. From band structures it can be observed that delocalized levels appear in $-0.90 \mathrm{eV}$ to $-0.29 \mathrm{eV}$ region due to an interaction between the aromatic ring of triclosan molecule and $(8,0)$ SWCNT (Fig. 2(a)). To elucidate in more detail the localization of the states in $-0.90 \mathrm{eV}$ to $-0.29 \mathrm{eV}$ region, the orbital charge density was obtained (Fig. 3(a)). It indicated a low contribution of orbitals of carbon atoms of SWCNT and higher contribution of orbitals of oxygen, carbon and chlorine atoms of triclosan molecule.

Differently from configuration of Fig. 1(a), in configurations (Fig. 1(b) and (c)), the band structure presented localized levels in valence band $0.6 \mathrm{eV}$ below Fermi level (see Fig. 2(b) and (c)). The plots of orbital charge density of these configurations, in $-0.63 \mathrm{eV}$ to $-0.29 \mathrm{eV}$ region, are shown in Fig. 3(b) and (c), respectively. It can be observed that larger contributions to these levels are of atoms from triclosan molecule. The band structure to configurations Fig. 1(d) and (e), are shown in Fig. 2(d) and (e), respectively. Both configurations presented similar electronic properties, for example, in both band structures the localized level appear about $0.86 \mathrm{eV}$ below the Fermi level and degenerated levels emerge below the Fermi level. The plots of orbital charge density between $-0.98 \mathrm{eV}$ and $-0.32 \mathrm{eV}$ show that levels are mixture the both SWCNT and triclosan orbitals (see Fig. 3(d) and (e)).

Regarding relaxed structure of the $(5,5)$ SWCNT the position of triclosan molecule, in relation to $(5,5)$ SWCNT are the same as considered to $(8,0)$ SWCNT. The minimum distance between $(5,5)$ nanotube and triclosan molecule is $3.12 \AA$ in the most stable configuration. All studied configurations exhibited energy binding lower than $0.83 \mathrm{eV}$ (Table 2), indicating that the interaction between triclosan molecule and $(5,5)$ SWCNT occurs through a physical process. In the preliminary analysis, the most stable configuration was the one in which the triclosan molecule is localized parallel to the $(5,5)$ tube axis. The positive values for binding energy indicate an attraction force between triclosan molecule and both SWCNT considered in this work.

From the values of charge transfer (Table 2), the triclosan molecule exhibited an electron donor character in the most favorable configuration energy and in configuration shown in Fig. 4(d).
Table 2

Calculated results for $E_{b}, D_{b}$ and CT to the attached triclosan molecule for the configurations of $(5,5)$ SWCNT shown in Fig. 4 . The minus and plus sign in the charge transfer values indicates that the triclosan molecule receives or donates electronic charge, respectively.

\begin{tabular}{llll}
\hline Configuration & $E_{b}(\mathrm{eV})$ & $D_{b} \AA$ & $\mathrm{CT}\left(\mathrm{e}^{-}\right)$ \\
\hline (a) & 0.53 & 3.12 & +0.03 \\
(b) & 0.26 & 2.29 & -0.11 \\
(c) & 0.30 & 2.13 & -0.12 \\
(d) & 0.10 & 3.20 & +0.01 \\
(e) & 0.28 & 2.97 & -0.05 \\
\hline
\end{tabular}

In the other configurations (Fig. 4(b), (c) and (e)) the triclosan molecule acts as acceptor electron.

Fig. 5(P) presents the band structure for the pristine (5,5) SWCNT to compared, while the Fig. 5(a)-(e) show the band structure to system presented in Fig. 4(a)-(e), respectively. From the band structures, it can be observed that pristine $(5,5)$ SWCNT behaved like a metallic system (see Fig. 5(P)). Moreover, a comparison between the electronic band structures of the pristine $(5,5)$ SWCNT with triclosan molecule adsorbed on the $(5,5)$ SWCNT showed no meaningful changes around the Fermi energy, even for configurations with the largest binding energies. All these results indicate a weak interaction between triclosan molecule and $(5,5)$ SWCNT.

In Fig. 6(a)-(e) the plots for the localized density of states (LDOS) of triclosan molecule adsorbed on the $(5,5)$ SWCNT for all configurations (shown in Fig. 4) are presented for the electronic levels located in region around $-1.07 \mathrm{eV}$ to $1.09 \mathrm{eV}$ (see Fig. 5(a)-(e)). From these LDOS plots, we observed that the largest contribution in this region belong to triclosan molecule, showing again a weak interaction between triclosan molecule and $(5,5)$ SWCNT.

\section{Conclusion}

In summary, we performed the first principles calculations on total energy, charge transfer, and electronic structures of the triclosan molecule interacting with semiconductor $(8,0)$ and metallic $(5,5)$ SWCNT. The configurations present mostly weak binding energies, which are typical of physical process. The one exception occurs in the case of interaction of the triclosan molecule on $(8,0)$ nanotube. This most stable configuration is the triclosan molecule 
parallel to the nanotube axis, which occur due the $\pi-\pi$ interactions between them. The semiconductor SWCNT present predominantly binding energies larger than that of metallic SWCNT, when comparing directly the same type configuration. Triclosan behaves as an electron donor or acceptor depending on configuration. These results show that carbon nanotubes are very promising for triclosan adsorption since it is sensible to presence of triclosan molecule and therefore could be used as a filter system for wastewater treatment.

\section{Acknowledgements}

We thank the CENAPAD-SP for the computer facilities. This work is partially supported by CNPq and FAPEMA. S.M. Castro acknowledges to CAPES for the fellowship.

\section{References}

[1] W. Chau, J. Wu, Z. Cai, Investigation of levels and fate of triclosan in environmental waters from the analysis of gas chromatography coupled with ion trap mass spectrometry, Chemosphere 73 (2008) S13-S17.

[2] S. Zhou, Y. Shao, N. Gao, J. Deng, C. Tan, Equilibrium, kinetic, and thermodynamic studies on the adsorption of triclosan onto multi-walled carbon nanotubes, Clean Soil Air Water 41 (2013) 539-547.

[3] M.G. Cantwell, B.A. Wilson, J. Zhu, G.T. Wallace, J.W. King, C.R. Olsen, R.M Burgess, J.P. Smith, Temporal trends of triclosan contamination in dated sediment cores from four urbanized estuaries: evidence of preservation and accumulation, Chemosphere 78 (2010) 347-352.

[4] C.T. Anger, C. Sueper, D.J. Blumentritt, K. McNeill, D.R. Engstrom, W.A. Arnold, Quantification of triclosan, chlorinated triclosan derivatives, and their dioxin photoproducts in lacustrine sediment cores, Environ. Sci. Technol. 47 (2013) 1833-1843.

[5] H.P. Schweizer, Triclosan: a widely used biocide and its link to antibiotics, FEMS Microbiol. Lett. 202 (2001) 1-7.

[6] A. Aguëra, A.R. Fernaández-Alba, L. Piedra, M. Mézcua, M.J. Gómez, Evaluation of triclosan and biphenylol in marine sediments and urban wastewaters by pressurized liquid extraction and solid phase extraction followed by gas chromatography mass spectrometry and liquid chromatography mass spectrometry, Anal. Chim. Acta 480 (2003) 193-205.

[7] Y. Dong, D.Y. Wu, X.C. Chen, Y. Lin, Adsorption of bisphenol A from water by surfactant-modified zeolite, J. Colloid Interface Sci. 348 (2010) 585-590.

[8] B. Pan, D.H. Lin, H. Mashayekhi, B.S. Xing, Adsorption and hysteresis of bisphenol A and $17 \alpha$-ethinyl estradiol on carbon nanomaterials, Environ. Sci. Technol. 43 (2009) 5480-5485.

[9] Y. Zhang, C. Causserand, P. Aimar, J.P. Cravedi, Removal of bisphenol A by a nanofiltration membrane in view of drinking water production, Water Res. 40 (2006) 3793-3799.

[10] G. Centi, S. Perathoner, Remediation of water contamination using catalytic technologies, Appl. Catal. B: Environ. 41 (2003) 15-29.

[11] H.D. Burrows, L.M. Canle, J.A. Santaballa, S. Steenken, Reaction pathways and mechanisms of photodegradation of pesticides, J. Photochem. Photobiol. B: Biol. 67 (2002) 71-108.
[12] S. Iijima, Synthesis of carbon nanotubes, Nature 354 (1991) 56-58.

[13] P. Chingombe, B. Saha, R.J. Wakeman, Surface modification and characterisation of a coal-based activated carbon, Carbon 43 (2005) 3132-3142.

[14] Y.H. Li, Z.C. Di, J. Ding, D.H. Wu, Z.K. Luan, Y.Q. Zhu, Adsorption thermodynamic, kinetic and desorption studies of $\mathrm{Pb}^{2}+$ on carbon nanotubes, Water Res. 39 (2005) 605-609.

[15] W. Chen, L. Duan, L.L. Wang, D. Zhu, Adsorption of hydroxyl- and amino-substituted aromatics to carbon nanotubes, Environ. Sci. Technol. 42 (2008) 6862-6868.

[16] C.S. Lu, Y.L. Chung, K.F. Chang, Adsorption of trihalomethanes from water with carbon nanotubes, Water Res. 39 (2005) 1183-1189.

[17] Q. Zaib, I.A. Khan, N.S. Saleh, J.R.V. Flora, Y.-G. Park, Y. Yoon, Removal of bisphenol $\mathrm{A}$ and $17 \beta$-estradiol by single-walled carbon nanotubes in aqueous solution: adsorption and molecular modeling, Water Air Soil Pollut. 223 (2012) 3281-3293.

[18] B. Pan, K. Sun, B. Xing, Adsorption kinetics of $17 \alpha$-ethinyl estradiol and bisphenol A on carbon nanomaterials. II. Concentration-dependence, J. Soils Sediments 10 (2010) 845-854.

[19] P. Hohenberg, W. Kohn, Inhomogeneous electron gas, Phys. Rev. 136 (1964) $864 \mathrm{~B}-871 \mathrm{~B}$

[20] P. Ordejon, J.M. Soler, Self-consistent order-N density-functional calculations for very large systems, Phys. Rev. B 53 (1996) 10441-10444.

[21] W. Kohn, L.J. Sham, Self-consistent equations including exchange and correlation effects, Phys. Rev. 140 (1965) A1133-A1138.

[22] E. Artacho, D. Sachez-Portal, P. Ordejon, A. Garcia, J.M. Soler, Linear-scaling ab-initio calculations for large and complex systems, Phys. Status Solidi B 215 (1999) 809-817.

[23] J.P. Perdew, A. Zunger, Self-interaction correction to density-functional approximations for many-electron systems, Phys. Rev. B 77 (1996) 5048-5079.

[24] N. Troullier, J.L. Martins, Efficient pseudopotentials for plane-wave calculations, Phys. Rev. B 43 (1991) 1993-2006.

[25] L. Kleinman, D.M. Bylander, Efficacious form for model pseudopotentials, Phys. Rev. Lett. 48 (1982) 1425-1428.

[26] H.J. Monkhorstand, J.D. Pack, Special points for Brillouin-zone integrations, Phys. Rev. B 13 (1976) 5188-5192.

[27] M.S. Dresselhaus, G. Dresselhaus, R. Saito, Physics of carbon nanotubes, Carbon 33 (1995) 883-891.

[28] M. Wessel, N.V. Skorodumova, Adhesion of the TiN/Fe interface with point defects from first principles, J. App. Phys. 113 (2013), 014905-014905-7.

[29] S. Guerini, A.G. Souza Fiho, J. Mendes Filho, O.L. Alves, S.B. Fagan, Electronic properties of $\mathrm{FeCl}_{3}$-adsorbed single-wall carbon nanotubes, Phys. Rev. B 72 (2005) 233401-233405.

[30] S.F. Boys, F. Bernardi, The calculation of small molecular interactions by the differences of separate total energies. Some procedures with reduced errors, Mol. Phys. 19 (1970) 553-566.

[31] F.M. Machado, C.P. Bergmann, T.H.M. Fernandes, E.C. Lima, B. Royer, T. Calvete, S.B. Fagan, Adsorption of Reactive Red M-2BE dye from water solutions by multi-walled carbon nanotubes and activated carbon, J. Hazard. Mater. 192 (2011) 1122-1131.

[32] F.M. Machado, C.P. Bergmann, E.C. Lima, B. Royer, F.E. Souza, I.M. Jauris, T. Calvete, S.B. Fagan, Adsorption of Reactive Blue 4 dye from water solutions by carbon nanotubes: experiment and theory, Phys. Chem. Chem. Phys. 14 (2012) 11139-11153. 